\title{
¿ES POSIBLE DAR EL PECHO SIN EMBARAZO PREVIO? PROTOCOLOS PARA LA INDUCCIÓN A LA LACTANCIA MATERNA
}

Is It Possible to Breastfeed without Previous Pregnancy? Protocols for Breastfeeding Induction

Montserrat Rocio Garrote Muñoz ${ }^{1}$, Pepita Giménez-Bonafé ${ }^{1}$

Autor correspondencia: Pepita Giménez-Bonafé

Correo electrónico: pgimenez@ub.edu

1. Unidad de Fisiología, Departamento de Ciencias Fisiológicas, Facultad de Medicina y Ciencias de la Salud, Campus de Bellvitge, Universitat de Barcelona. (L'Hospitalet de Llobregat, España)

Recibido: 19/06/2021 Aceptado: 29/12/2021 


\section{RESUMEN}

INTRODUCCIÓN. La lactancia inducida es un método a través del cual una madre no gestante puede dar el pecho a su bebé. Los nuevos modelos de familia formados por parejas homosexuales, gestación por sustitución y adopción son los casos típicos en los que se puede optar por llevar a cabo este proceso. Los métodos existentes para la inducción son farmacológicos y no farmacológicos, ambos recogidos en unos protocolos específicos. OBJETIVOS. Profundizar sobre el mecanismo que permite la inducción de la lactancia, los tipos de protocolos existentes, detallando qué técnicas se usaron y determinando cuáles son los factores de éxito para una inducción satisfactoria. MATERIAL Y MÉTODOS. Búsqueda bibliográfica en las siguientes bases de datos: Pubmed, Web of Science, Cinahl. Se amplía la búsqueda en Journal of Human Lactation y la revista Musas. Fueron seleccionados 16 artículos de casos relacionados con la inducción a la lactancia. RESULTADOS. La mayoría de las madres consiguen instaurar la lactancia gracias a la motivación personal, el apoyo emocional y la estimulación y succión del pecho. CONCLUSIONES. Surge la necesidad de informar a los sanitarios y consultores para que promuevan y asesoren sobre la lactancia inducida. Son necesarios más estudios para poder estandarizar los métodos inductivos y poderlos individualizar en función del caso, objetivos y expectativas, ya que, en función de estos, se determinará el éxito de la inducción.

Palabras clave: inducción a la lactancia; adopción, homosexualidad; subrogación; galactogogos.

\section{ABSTRACT}

INTRODUCTION. Induced lactation is a method by which a non-pregnant mother can breastfeed her baby. New family models such as homosexual couples, surrogate gestation and adoption are the typical cases in which you can choose to carry out this process. The existing methods for induction are pharmacological and non-pharmacological, both included in specific protocols.

OBJECTIVES. To delve into the mechanism that allows lactation induction, the types of existing protocols, detailing what techniques were used and determining what are the success factors for successful induction. MATERIAL AND METHODS. Bibliographic research in the following databases: Pubmed, Web of Science, Cinahl. The search is expanded in the Journal of Human Lactation and Musas. Sixteen case articles related to lactation induction were selected. RESULTS. Most mothers manage to establish lactation thanks to personal motivation, emotional support, breast stimulation and sucking. CONCLUSIONS. There is a need to inform health professionals and consultants so that they can promote and advise on induced lactation when needed. More studies are necessary to be able to standardize inductive methods and to be able to individualize them according to the case, objectives, and expectations, since, based on these, the success of the induction will be determined.

Keywords: Induced Lactation; Adoption; Homosexuality; Subrogation; Galactogogues. 


\section{INTRODUCCIÓN}

La lactancia materna exclusiva es el método de alimentación que se recomienda durante al menos los primeros seis meses de vida (OMS, 2017). Este alimento le proporciona al lactante beneficios a nivel inmunológico y metabólico para su correcto crecimiento y protección frente a numerosas enfermedades crónicas e infecciosas. Así mismo, ofrece un mejor desarrollo neurocognitivo, una menor morbimortalidad y una disminución de la muerte súbita del lactante (Kasper et al., 2018). Los efectos en la madre son una reducción del riesgo de cáncer de mama y ovario, diabetes tipo 2 y depresión postparto (Kasper et al., 2018; OMS, 2017). En lo que respecta a ambos, madre y bebé, la lactancia promueve un fuerte vínculo emocional entre ellos (Gribble, 2007). De hecho, socialmente la lactancia se ve como parte del proceso y transición que implica el hecho de ser madre (Marshall et al., 2007; Williams et al., 2013). Este simbolismo de unión maternofilial, junto con las actuales tendencias de «maternidad intensiva», cuyos objetivos se basan en proporcionar al bebé lo mejor en relación con cuidados, alimentación, necesidades y deseos sobreponiendo el bienestar del bebé por encima del de la madre, pueden influir en la decisión de dar el pecho (Afflerback et al., 2013; Cappellini et al., 2019).

Tradicionalmente, el suceso fisiológico de amamantar se asocia a madres biológicas, pero no solo se reserva esta capacidad a ellas, pues a través de un proceso de inducción de la lactancia, madres no gestantes pueden dar el pecho. Esto no es un concepto nuevo, pero bien es cierto que, en la actualidad, a través del cambio de modelos de familia que experimentamos en nuestra sociedad a través de las adopciones, la gestación subrogada o de sustitución y las parejas de dos madres, ha ganado cierto renombre y puede ser una opción más.

El proceso mediante el cual una madre que no ha estado embarazada nunca ni ha dado previamente el pecho consigue producir leche y amamantar se denomina «inducción a la lactancia». Por el contrario, en madres que ya han dado el pecho con anterioridad, se puede reestablecer la lactancia y este proceso recibe el nombre de «relactación» (OMS, 1998).

Los motivos por los cuales se induce la lactancia difieren según el país. En países no occidentales, el objetivo fundamental es la supervivencia del bebé. Casos donde el lactante esté enfermo, malnutrido, o madres a las que les es imposible amamantar por enfermedad, como por ejemplo el VIH, o por su fallecimiento, son circunstancias de las 
que se podrían servir el proceso de la inducción o relactación por parientes cercanos o de la misma comunidad, normalmente las abuelas (Ongulesi et al., 2008).

En países occidentales, la finalidad de la inducción de la lactancia materna es la creación del vínculo emocional entre madre e hijo. Según la teoría del apego de Bowly, tanto la madre como el bebé necesitan mantenerse en contacto y crear ese vínculo o apego para el correcto desarrollo en la esfera física, emocional y mental y la supervivencia del bebé (Bowlby, 1996). Así pues, el motivo principal en estos países es proporcionar beneficios a nivel emocional, crear y fortalecer el vínculo del binomio «madre-bebé», promoviendo esa proximidad y contacto entre ambos (Cazorla-Ortiz et al., 2019; Gribble, 2007).

En España, existen casos de inducción a la lactancia en parejas de gestación por sustitución, adopción o parejas de dos madres, pero la prevalencia de familias que llevan a cabo este proceso es desconocida. De hecho, es inexistente una base de datos al respecto (Cazorla-Ortiz et al., 2019). No obstante, la popularidad con respecto al tema de la inducción está ganando terreno a través del cambio de las estructuras y modelos familiares descritos. Por lo que a términos de lactancia en general se refiere, sí se tienen datos de prevalencia, situando a España en su último informe anual del Sistema Nacional de Salud con un porcentaje del $73,9 \%$ de lactantes que hasta la sexta semana de vida son alimentados con leche materna exclusiva. Este porcentaje disminuye en lactantes de 3 meses hasta un 63,9\%, y hasta un 39\% en lactantes de 6 meses (Ministerio de Sanidad, Servicios Sociales e Igualdad, 2019).

Así pues, uno se podría preguntar sobre la posibilidad de amamantar a su hijo/a con su propia leche materna sin la necesidad de haber estado embarazada. ¿Es posible dar el pecho sin embarazo previo? El objetivo de esta revisión es profundizar sobre los mecanismos que permiten la inducción de la lactancia, los tipos de protocolos y técnicas existentes, y describir cuáles son los factores de éxito para lograr tener una inducción satisfactoria.

\section{MATERIAL Y MÉTODOS}

Se realiza una revisión bibliográfica entre los meses de diciembre 2019 y febrero de 2020 en las siguientes bases de datos: Pubmed, Web of Science, Cinahl. Se amplía la búsqueda en Journal of Human Lactation y la revista Musas. Revista de investigación en mujer, salud y sociedad. 
Las palabras de lenguaje libre usadas como descriptores fueron «induced lactation», «inducing lactation», «relactation», «breastfeeding», «lactation» $\mathrm{y}$ «human milk».

En cuanto a las combinaciones boleanas, se usaron «induced lactation or inducing lactation», «relactation and HIV», «induced lactation and adoption», «lactation and adoption», «induced lactation and homosexuality», «lactation and homosexuality», «induced lactation and surrogate mothers», «lactation or human milk and adoption», «lactation or breastfeeding or human milk and adoption» $\mathrm{y}$ «lactation or breastfeeding or human milk and adoption or female sexuality or surrogate mothers».

Criterios de inclusión: Por motivos de escasez de literatura y por la importancia y trascendencia de los artículos publicados en los años ochenta, se ha incluido la evidencia existente acerca del tema de la inducción a la lactancia desde entonces hasta la actualidad.

Criterios de exclusión: estudios que no fuesen relacionados con la inducción a la lactancia, estudios en animales y artículos escritos en otros idiomas que no fuesen español o inglés.

Figura 1. Diagrama de flujo de los artículos seleccionados

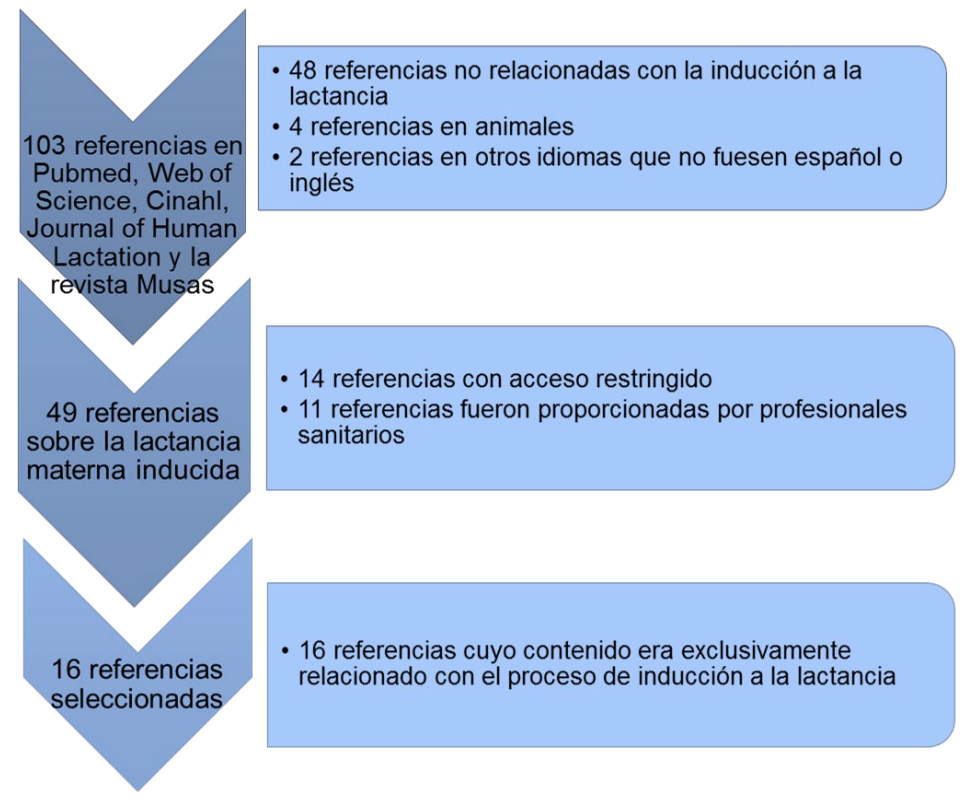

Por la escasez de publicaciones, una vez evaluada su calidad mediante la guía Prisma, se decidió incluirlas todas. Del total de 103 artículos, fueron seleccionados 16 (Figura 1), ya que el resto no incluía información sobre la lactancia inducida, eran estudios en animales o estaban escritos en idiomas distintos al español e inglés. Los artículos que se seleccionaron, a través de una revisión manual, contienen información sobre el proceso de inducción a la 
lactancia, métodos utilizados y si se pudo amamantar o no. En la selección se tuvo en cuenta también, con respecto a los artículos clásicos, el grado de impacto que tuvieron sentando las bases para los siguientes estudios que se realizaron años después revisando manualmente cuántos y cuáles de ellos los citaban.

\section{RESULTADOS}

\section{MÉTODOS DE INDUCCIÓN Y PROTOCOLOS}

\subsection{Inducción farmacológica}

Jack Newman y Lenore Goldfarb sientan las bases de este procedimiento (2002), cuyo objetivo principal es imitar la situación fisiológica hormonal propia del embarazo que induce los cambios necesarios en el pecho preparándolo para la lactancia, y la situación hormonal postparto que promueve la producción y eyección de leche. Esto se consigue mediante la toma de una píldora anticonceptiva que contiene estrógenos y progesterona, juntamente con la toma de Domperidona.

\section{Domperidona y otros galactogogos}

Antagonista dopaminérgico generalmente usado como antiemético. Como efectos adversos, puede aumentar los niveles de prolactina pudiendo inducir la lactancia. Por ello se le considera un galactogogo o fármaco inductor de la producción de leche. Las concentraciones de Domperidona que pueden resultar en la producción de leche varían en función de la dosis, con lo cual, para su uso como galactogogo, se sugiere una dosis de 10-20 mg vía oral 3-4 veces al día. Los efectos extrapiramidales son muy infrecuentes y no atraviesa la barrera hematoencefálica, con lo que no presenta efectos neurológicos y resulta más segura para la madre y bebé. De hecho, es catalogado como un fármaco seguro, compatible con la lactancia y con un riesgo muy bajo para el lactante, siendo ampliamente usado como galactogogo. Un hecho a remarcar sería que se asocia con un incremento del riesgo de padecer arritmias, especialmente en personas mayores, con patologías cardíacas previas o con el uso concomitante de otros fármacos que alargan el QT. Es extremadamente infrecuente que pueda suceder en una mujer sana sin patologías previas, pero se ha de tener en cuenta a la hora de su prescripción (Apilam, 2002).

La Metoclopramida sería un fármaco similar. También es un antiemético y antagonista dopaminérgico, así como un galactogogo y fármaco seguro con riesgo muy bajo para la lactancia y el lactante. No obstante, este sí atraviesa la barrera 
hematoencefálica, pudiendo causar efectos adversos a nivel neurológico, como síntomas extrapiramidales o depresión, con lo que se prefiere la Domperidona para dicha finalidad (Apilam, 2002).

Otros fármacos también descritos como galactogogos serían la Clorpromazina y la Sulpirida. La Clorpromazina, ansiolítico, antipsicótico y antiemético también puede, al inhibir la dopamina, aumentar los niveles de prolactina y aumentar la producción de leche. No obstante, se considera de riesgo bajo para la lactacia con riesgo leve para el lactante, con posibles, aunque poco probables, efectos sedantes (Apilam, 2020). La Sulpirida es un antipsicótico, antidepresivo, antiemético y procinético antagonista selectivo de la dopamina que aumenta también los niveles de prolactina, siendo usado así, pues, como galactogogo. Tiene un riesgo muy bajo para la lactancia, pero aunque no esté publicada, existe la probabilidad de efectos en el lactante del tipo psicotrópicos, y de síntomas extrapiramidales y arritmias en la madre. Por estos posibles efectos, hay quienes no consideran el uso adecuado de la Clorpromazina y especialmente de la Sulpirida para inducir la lactancia (Apilam, 2002).

Finalmente, la utilización de oxitocina vía spray intranasal o el uso de la hormona liberadora de tiroxina son más ejemplos de fármacos facilitadores de la extracción láctea y galactogogos respectivamente, pero menos ampliamente disponibles, utilizados y descritos (Hormann et al., 1998).

\section{Píldora anticonceptiva}

La píldora ha de contener $1 \mathrm{mg}$ de progesterona (preferible si son 2-3 mg) y no más de $0,035 \mathrm{mg}$ de estrógeno. Se inicia su toma en cualquier punto del ciclo menstrual y ha de ser tomada sin interrupciones el tiempo indicado según el protocolo establecido. Por estos dos motivos la píldora no resulta eficaz como método anticonceptivo, pero sí para favorecer el desarrollo mamario necesario para dar el pecho, con lo cual se recomiendan el uso de otros métodos para evitar la concepción (Goldfarb et al., 2002).

Es importante recalcar que, estos fármacos deben ser prescritos y supervisados por un profesional sanitario.

\subsection{Inducción no farmacológica}

Este método de inducción se basa en el uso de las hierbas como galactogogos (fitoterapia) y/o la estimulación del pecho y pezón manualmente y con el uso de un sacaleches. 


\section{Hierbas}

Es conocido el hecho de que el consumo de ciertas hierbas y plantas puede ser de ayuda para la producción de leche. Entre estas se encuentran principalmente el Fenogreco y el Cardo Bendito y se recomienda tomar 3 cápsulas de cada una 3 veces al día. Si se desea el uso conjunto con un método farmacológico, como la toma de la píldora anticonceptiva, se recomienda iniciar la fitoterapia una vez finalizada la prescripción de esta (Goldfarb y Newman, 2002). Otros galactogogos naturales conocidos son la levadura de cerveza, la moringa, el hinojo y el Cardo Mariano. Todos los citados son considerados de riesgo muy bajo para la lactancia, siendo seguros y compatibles con esta, a excepción del hinojo que es catalogado de poco seguro con alto riesgo para la lactancia por sus efectos neurotóxicos (Apilam, 2002).

Cabe decir que el uso en exceso de estos productos puede provocar intoxicaciones e interaccionar con otros fármacos. Con respecto a la literatura acerca del tema, existen publicaciones sobre su efectividad, aunque la evidencia científica con respecto a la seguridad y eficacia real de las hierbas usadas como galactogogos es escasa (Cazorla Ortiz, 2018; Hormann et al., 1998; Mortel et al., 2013).

\section{Estimulación del pecho y sacaleches}

Durante el proceso de inducción, es el factor más importante y puede realizarse mediante el uso combinado de masajes y extracciones frecuentes preferiblemente con un sacaleches eléctrico (mejor si es doble) o, en su defecto, con un sacaleches manual (Cazorla Ortiz, 2018; Flores-Antón et al., 2017; Goldfarb y Newman, 2002).

Todos los métodos descritos pueden encontrarse en la Figura 2, en la que se explica un algoritmo para conseguir la inducción de la lactancia, según el tipo de inducción deseado.

Existen distintos protocolos para la inducción de la lactancia, planteados de nuevo por Jack Newman y Lenore Goldfarb (2002), basados en los métodos descritos.

\subsection{Protocolo Regular}

Sugerido para aquellas madres que tengan de margen mínimo 6 meses antes del nacimiento o llegada de su bebé.

6 meses antes de la llegada del bebé, se inicia la toma de una píldora anticonceptiva por día juntamente con la toma de Domperidona $10 \mathrm{mg} 4$ veces al día durante la primera 
semana. Pasada la primera semana, se incrementa la dosis de Domperidona a $20 \mathrm{mg} 4$ veces al día y se mantiene esta combinación hasta 6 semanas antes de la llegada del bebé. A partir de entonces, se para la píldora anticonceptiva continuándose la Domperidona con la misma posología. Parando la píldora, ante la bajada hormonal, la madre debe experimentar sangrado vaginal, de lo contrario, y en caso de ser fértil, debería estudiarse un posible embarazo. Las siguientes semanas hasta la llegada del bebé la madre ha de realizar la estimulación de pecho junto con extracciones. Se recomiendan primeramente masajes en el pecho, incluyendo areola y pezón, así como realizar suaves tracciones sobre este. Las extracciones son preferibles con sacaleches eléctrico doble, cada 3 horas durante el día y cada 4 horas durante la noche, con una duración de unos 5-7 minutos. Es importante la extracción nocturna dado que, de forma fisiológica en la madre, la prolactina aumenta entre la 1 y las 5 de la madrugada, con lo que, si aprovechamos este hecho, junto con el hecho de que cuántas más extracciones mayor producción de leche, el resultado será mucho más favorable (Goldfarb y Newman, 2002).

Este proceso intenta imitar la fisiología como bien se comentó con anterioridad (Goldfarb y Newman, 2002), de manera que mantenemos en una primera fase, gracias a la píldora, niveles hormonales elevados de estrógeno y progesterona simulando el embarazo que ayudan a preparar al pecho para que, cuando la paremos y continuemos con la Domperidona y empecemos con la estimulación, entremos en una segunda fase simulando el postparto donde el descenso hormonal de progesterona y el estímulo de succión induce una subida de los niveles de prolactina y oxitocina para que se pueda dar lugar a la producción y eyección de leche.

Cuando se empieza la estimulación, se puede añadir la ingesta de hierbas galactogogas, como el Fenogreco y el Cardo Bendito mencionados anteriormente, tomando 3 cápsulas de cada una de ellas 3 veces al día junto con las comidas. Las cápsulas han de contener aproximadamente $610 \mathrm{mg}$ de Fenogreco y $390 \mathrm{mg}$ de Cardo Bendito.

La aparición de leche suele ser al principio en forma de gotas que luego, con el tiempo, darán lugar a un chorro eyectivo de leche. El tiempo que transcurre entre que se inicia la estimulación y se logra extraer leche puede ser desde días a semanas, dado que cada cuerpo es distinto y por ello responde de forma diferente (Goldfarb y Newman, 2002). 
Una vez tiene lugar la llegada del bebé, se debe continuar con la misma posología de Domperidona y poner, tan pronto como sea posible, el bebé al pecho y establecer una lactancia a demanda. En un primer momento, se recomienda al final de cada toma realizar una extracción con el sacaleches de unos 10 minutos para favorecer el aumento de producción. Las hierbas también se pueden seguir consumiendo hasta que la lactancia esté bien establecida. Una vez lleguemos a este punto, se podría empezar a reducir la dosis de Domperidona gradualmente e incluso llegar a dejar de tomarla (Goldfarb y Newman, 2002).

\subsection{Protocolo acelerado}

Este protocolo se ajustaría a aquellas madres que disponen de menos de 6 meses antes de la llegada de su bebé. Para estas circunstancias, la píldora anticonceptiva se toma durante 30-60 días sin interrupciones junto con la Domperidona a dosis directamente de $20 \mathrm{mg} 4$ veces al día. Si la madre percibe cambios en el pecho, tales como incremento del tamaño de al menos una copa, sensación de plenitud, pesadez y dolor en los primeros 30 días, se detiene la toma de la píldora manteniendo la ingesta de Domperidona e iniciando la estimulación del mismo modo que en el protocolo regular.

La producción de leche puede ser menor en comparación con el protocolo regular, pero no obstante suele ser suficiente para abastecer las necesidades del bebé. Se podrían utilizar métodos de suplementación si fuese necesario.

En caso de que la madre disponga de 4 semanas o menos, o incluso si decide dar el pecho una vez llega el bebé, la inducción es posible. Se empieza la toma de la píldora anticonceptiva junto con los $20 \mathrm{mg}$ de Domperidona 4 veces al día. Lo ideal sería que la madre pudiese tomar la píldora al menos 30 días y una vez completado este tiempo se detendría su uso manteniendo la Domperidona e iniciando, con sacaleches eléctrico doble, la estimulación. Del mismo modo que en el protocolo regular, una vez se inicia la estimulación, se pueden añadir las hierbas galactogogas con la posología descrita.

Los diferentes tipos de protocolos de inducción a la lactancia dependiendo del tiempo disponible se reflejan en el algoritmo de la Figura 2.

\subsection{Protocolo para mujeres menopáusicas}

Está indicado para mujeres con menopausia como estado fisiológico o como consecuencia debido a una cirugía ginecológica. Primeramente, en caso de que la madre 
se encuentre con terapia hormonal sustitutiva, se debe parar y reemplazar por la píldora anticonceptiva e iniciar Domperidona de forma gradual como en el protocolo regular. Se mantiene dicha combinación hasta que experimenta la madre los cambios en pecho descritos en el protocolo acelerado. Al menos se recomienda, para mujeres menopáusicas, un mínimo de 60 días con ambos fármacos. Una vez completado ese mínimo y se hayan experimentado los cambios en el pecho, se para la anticonceptiva, se continua con Domperidona y se empieza la estimulación, pudiendo añadir el uso de hierbas, del mismo modo que en los protocolos anteriores.

\subsection{Condiciones especiales}

En caso de que la madre tenga antecedentes de trombosis, patología cardíaca o hipertensión arterial, se contraindica el uso de la píldora anticonceptiva. No obstante, se han dado casos de madres donde subministrando el antihipertensivo Metildopa, han respondido bien y se ha permitido el uso concomitante con la píldora, beneficiándose incluso en un mayor incremento de producción de leche, ya que este fármaco aumenta los niveles de prolactina (Apilam, 2002; Goldfarb y Newman, 2002).

La Sertralina suele ser un fármaco eficaz en caso de que la madre presente antecedentes de depresión, siendo compatible con la lactancia y, probablemente, el antidepresivo más seguro en estas condiciones (Apilam, 2002; Goldfarb y Newman, 2002).

En cuanto a la píldora anticonceptiva, existen mujeres que no la toleran. En estos casos, se pueden intentar alternativas tales como estrógenos y progesterona tópicos en forma de cremas o parches, junto a la ingesta de Domperidona. Incluso en esta situación, la madre podría optar por la toma de únicamente Domperidona junto a hierbas. Existen otras mujeres que no experimentan los cambios en el pecho descritos en los protocolos mientras toman la píldora. La solución en estos casos sería o bien sustituir la píldora por otra que contenga más cantidad de progesterona, o bien continuar con la misma y añadir dosis extra de progesterona tomando otra píldora con dosificación más alta de esta hormona (Goldfarb y Newman, 2002).

Finalmente, respecto a las hierbas como el Fenogreco propuesto, se ha de tener en cuenta que puede tener un efecto hipoglucemiante, con lo cual, en madres diabéticas, debe emplearse con mucha precaución. De la misma forma, el Fenogreco podría agravar los 
síntomas respiratorios típicos del asma, así que también debe ser tomado con precaución en madres con antecedentes asmáticos (Goldfarb y Newman, 2002).

\subsection{Composición de la leche resultado de la inducción}

Existen estudios que han comparado la composición de la leche materna de madres gestantes y de madres no gestantes que han llevado a cabo un proceso de inducción. Los resultados concluyen que la leche resultado de la inducción contiene unos niveles parecidos o incluso mayores en cuanto al total de proteínas, anticuerpos tipo $\operatorname{IgA}$, lactoferrina y lisoenzimas, comparada con la leche de madres que fisiológicamente han pasado por un embarazo. Por tanto, no se encuentran diferencias significativas entre ambas composiciones. No obstante, se proponen estudios acerca de la leche inducida conforme más aumenta su producción y de la leche extraída mediante sacaleches o por la succión del bebé para ver si existe alguna diferencia (Perrin et al., 2015).

\section{FACTORES DE ÉXITO Y DIFICULTADES QUE LAS MADRES PUEDEN EXPERIMENTAR}

Hay que tener en mente que lo más importante para las madres, como bien se ha citado en el apartado «Finalidad de la inducción de la lactancia», es crear un vínculo con su bebé, con lo que han de tener apoyo a nivel emocional por parte de su entorno personal y sanitario (Gribble, 2004) que ayude a afianzar la confianza en ellas mismas y la motivación para poder llevar a cabo este proceso con las dificultades que pueden aparecer (Cazorla Ortiz et al., 2019).

Otro requerimiento para una inducción favorable y exitosa es la frecuente y adecuada estimulación del pecho antes de la llegada del bebé y la succión de este a posteriori (Flores-Antón et al., 2017). El hecho de que el bebé esté dispuesto a mamar puede depender de su disposición y habilidad, de su edad, del método de alimentación y tiempo transcurrido entre el nacimiento y la llegada a su nueva familia, y de condiciones que le impidan mamar bien, como por ejemplo un mal agarre o la presencia de frenillo lingual (Hormann et al., 1998; Ministerio de Sanidad, Servicios Sociales e Igualdad, 2017).

En cuanto a las dificultades que afrontan estas mujeres, suelen ser la falta de información acerca del tema, la falta de soporte por parte de profesionales sanitarios debido al desconocimiento del tema y la incertidumbre de si podrán ser capaces o no de dar el pecho. Por ello mismo, las madres que se adentran en este proceso pueden llegar 
a sentir miedo, inseguridad o estrés, de manera que el soporte y la confianza en ellas es crucial (Flores-Antón et al., 2017).

En España, un estudio realizado a madres que han inducido la lactancia pone de manifiesto dichas dificultades citadas y se añade, en cuanto a dificultades que provienen propiamente del hecho de amamantar, la cantidad de leche que son capaces de producir. No obstante, que algunas madres no pudiesen lograr la lactancia materna exclusiva no les supuso un impacto negativo dado que su principal objetivo era sentir esa proximidad con su bebé (Cazorla Ortiz et al., 2019).

También se añade una mayor dificultad en aquellas mujeres que inducen la lactancia en comparación con aquellas que relactan (Cazorla Ortiz et al., 2019). Esto podría sustentarse en otro estudio que informa acerca de la capacidad de la glándula mamaria de reaccionar de una forma más exacerbada a una segunda gestación. Se demuestra en glándulas mamarias de ratón que este hecho es consecuencia de una memoria epigenética a largo plazo del embarazo y su efecto en la mama. Concretamente, la primera gestación induce cambios epigenéticos que promueven la rápida expresión de determinados genes en una siguiente gestación, resultando en una respuesta funcional mayor y más rápida de la glándula mamaria (Dos Santos et al., 2015).

\section{Resultados de la inducción a la lactancia según el tipo de pareja}

Tras el análisis de los 16 artículos seleccionados, a continuación se exponen unas tablas resumen (Tablas 1, 2, 3 y 4), dividiéndolas por el tipo de pareja que se somete al proceso de inducción de la lactancia, e incluyendo por tabla la siguiente información: autor y año de publicación, el tipo de estudio, los métodos que usaron para la inducción y si esta fue exitosa en cuanto a si se pudo dar el pecho o no y el tipo de lactancia obtenida. 
Tabla 3.1. Madres de adopción

\begin{tabular}{|c|c|c|c|}
\hline $\begin{array}{c}\text { Autor/es, año y } \\
\text { país }\end{array}$ & $\begin{array}{l}\text { Diseño del } \\
\text { estudio }\end{array}$ & Método de inducción & Resultados \\
\hline $\begin{array}{l}\text { Auerbach et al. } \\
\text { (1981) EEUU }\end{array}$ & Descriptivo & $\begin{array}{l}\text { Estimulación pecho y pezón } \\
\text { (manual +/- mecánica + succión } \\
\text { del bebé) + suplementación } \\
\text { dietética (vitamina B y abundante } \\
\text { ingesta de líquidos). Algunas } \\
\text { madres usaron hormonas. }\end{array}$ & $\begin{array}{l}\text { Satisfactorio. } \\
\text { Mayoría: Lactancia } \\
\text { mixta. }\end{array}$ \\
\hline $\begin{array}{l}\text { Nemba (1994) } \\
\text { Papúa Nueva } \\
\text { Guinea }\end{array}$ & $\begin{array}{l}\text { Estudio de } \\
\text { un caso }\end{array}$ & $\begin{array}{l}\text { Fármacos galactogogos }+ \\
\text { estimulación (succión bebé). } \\
\text { Algunas madres recibieron } \\
\text { progesterona. }\end{array}$ & $\begin{array}{l}\text { Satisfactorio. } \\
\text { Mayoría: Lactancia } \\
\text { materna exclusiva }\end{array}$ \\
\hline $\begin{array}{l}\text { Abejide et al. } \\
\text { (1997) Nigeria }\end{array}$ & $\begin{array}{l}\text { Estudio de } \\
\text { un caso }\end{array}$ & Succión del bebé & $\begin{array}{l}\text { Satisfactorio. } \\
\text { Lactancia materna } \\
\text { exclusiva }\end{array}$ \\
\hline $\begin{array}{l}\text { Cheales- } \\
\text { Siebenaler } \\
\text { (1999) EEUU }\end{array}$ & $\begin{array}{l}\text { Estudio de } \\
\text { un caso }\end{array}$ & $\begin{array}{l}\text { Metoclopramida }+ \text { oxitocina } \\
\text { intranasal en spray }+ \text { estimulación } \\
\text { (sacaleches eléctrico) }\end{array}$ & $\begin{array}{l}\text { Satisfactorio. } \\
\text { Lactancia materna } \\
\text { exclusiva y } \\
\text { posteriormente mixta }\end{array}$ \\
\hline $\begin{array}{l}\text { Szucs et al. } \\
\text { (2010) EEUU }\end{array}$ & $\begin{array}{l}\text { Estudio de } \\
\text { un caso }\end{array}$ & $\begin{array}{l}\text { Anticonceptivos orales }+ \\
\text { Domperidona }+ \text { estimulación } \\
\text { (sacaleches eléctrico doble) }+ \\
\text { fitoterapia (fenogreco y cardo } \\
\text { bendito) }\end{array}$ & $\begin{array}{l}\text { Satisfactorio. } \\
\text { Lactancia materna } \\
\text { exclusiva para ambos } \\
\text { gemelos prematuros. }\end{array}$ \\
\hline $\begin{array}{l}\text { Flores-Antón et } \\
\text { al. (2017) } \\
\text { Madrid }\end{array}$ & $\begin{array}{l}\text { Estudio de } \\
\text { un caso }\end{array}$ & $\begin{array}{l}\text { Domperidona }+ \text { estimulación } \\
\text { (sacaleches eléctrico doble y } \\
\text { extracción manual) }\end{array}$ & $\begin{array}{l}\text { Satisfactorio. Se } \\
\text { convierte en donante } \\
\text { de leche. }\end{array}$ \\
\hline
\end{tabular}


Tabla 3.2. Pareja de madres homosexuales

\begin{tabular}{|c|c|c|c|}
\hline $\begin{array}{l}\text { Autor/es, } \\
\text { año y país }\end{array}$ & $\begin{array}{l}\text { Diseño del } \\
\text { estudio }\end{array}$ & Método de inducción & Resultados \\
\hline $\begin{array}{l}\text { Wahlert et al. } \\
(2013) \\
\text { EEUU }\end{array}$ & $\begin{array}{l}\text { Caso de } \\
\text { ética }\end{array}$ & $\begin{array}{l}\text { Anticonceptivos }+ \\
\text { Metoclopramida o } \\
\text { Clorpromazina }+ \\
\text { estimulación (sacaleches } \\
\text { eléctrico) }+/ \text { - hierbas } \\
\text { galactogogas }\end{array}$ & $\begin{array}{l}\text { Resalta que los riesgos que } \\
\text { pudiese suponer la inducción } \\
\text { a la lactancia (mínimos) son } \\
\text { infinitamente superados por } \\
\text { los beneficios que supone } \\
\text { para el bebé y la relación } \\
\text { mamá-bebé. También pone } \\
\text { de manifiesto el } \\
\text { desconocimiento del tema por } \\
\text { parte de la obstetra. }\end{array}$ \\
\hline $\begin{array}{l}\text { Wilson et al. } \\
(2015) \\
\text { EEUU }\end{array}$ & $\begin{array}{l}\text { Estudio de } \\
\text { un caso }\end{array}$ & $\begin{array}{l}\text { Píldora anticonceptiva }+ \\
\text { Domperidona }+ \text { estimulación } \\
(\text { sacaleches eléctrico) }+ \\
\text { hierbas galactogogas } \\
\text { (Fenogreco y Moringa } \\
\text { Oleifera) }\end{array}$ & $\begin{array}{l}\text { Satisfactorio. Lactancia } \\
\text { materna mixta por parte de } \\
\text { ambas madres para su bebé } \\
\text { adoptado. }\end{array}$ \\
\hline $\begin{array}{l}\text { McGuire } \\
\text { (2019) } \\
\text { Australia }\end{array}$ & $\begin{array}{l}\text { Estudio de } \\
\text { un caso }\end{array}$ & $\begin{array}{l}\text { Píldora anticonceptiva }+ \\
\text { Domperidona }+ \text { estimulación } \\
(\text { sacaleches eléctrico) }\end{array}$ & $\begin{array}{l}\text { Satisfactorio. Lactancia } \\
\text { materna exclusiva compartida } \\
\text { entre la madre que se induce } \\
\text { y la madre biológica. }\end{array}$ \\
\hline
\end{tabular}


Tabla 3.3. Madres de bebés gestados por sustitución

\begin{tabular}{|c|c|c|c|}
\hline $\begin{array}{l}\text { Autor/es, año } \\
\text { y país }\end{array}$ & $\begin{array}{l}\text { Diseño del } \\
\text { estudio }\end{array}$ & Método de inducción & Resultados \\
\hline $\begin{array}{l}\text { Banapurmath } \\
\text { et al. (1993) } \\
\text { India }\end{array}$ & $\begin{array}{l}\text { Estudio de } \\
\text { un caso }\end{array}$ & $\begin{array}{l}\text { Metoclopramida }+ \\
\text { estimulación (masaje y } \\
\text { succión del bebé) }\end{array}$ & $\begin{array}{l}\text { Las mujeres consiguen } \\
\text { lactancia materna (algunas } \\
\text { exclusiva y otras mixta). }\end{array}$ \\
\hline $\begin{array}{l}\text { Biervliet et al. } \\
\text { (2001) } \\
\text { Reino Unido }\end{array}$ & $\begin{array}{l}\text { Estudio de } \\
\text { un caso }\end{array}$ & $\begin{array}{l}\text { Metoclopramida }+ \\
\text { estimulación (sacaleches } \\
\text { eléctrico) }\end{array}$ & $\begin{array}{l}\text { Satisfactorio. Lactancia } \\
\text { mixta. }\end{array}$ \\
\hline $\begin{array}{l}\text { Shiva et al. } \\
\text { (2010) Irán }\end{array}$ & $\begin{array}{l}\text { Estudio de } \\
\text { un caso }\end{array}$ & $\begin{array}{l}\text { Metoclopramida }+ \\
\text { estimulación (sacaleches } \\
\text { eléctrico) }\end{array}$ & Satisfactorio. Lactancia mixta \\
\hline $\begin{array}{l}\text { Farhadi et al. } \\
\text { (2017) } \\
\text { Irlanda }\end{array}$ & $\begin{array}{l}\text { Estudio de } \\
\text { un caso }\end{array}$ & $\begin{array}{l}\text { Imaginación }+ \text { estimulación } \\
\text { táctil del pecho }+ \\
\text { Metoclopramida }+ \text { sacaleches } \\
\text { eléctrico doble }\end{array}$ & Satisfactorio. Lactancia mixta \\
\hline $\begin{array}{l}\text { Zingler et al. } \\
\text { (2017) Brasil }\end{array}$ & $\begin{array}{l}\text { Estudio de } \\
\text { un caso }\end{array}$ & $\begin{array}{l}\text { Domperidona }+ \text { sacaleches } \\
\text { eléctrico }+ \text { succión bebé }\end{array}$ & Satisfactorio. Lactancia mixta \\
\hline
\end{tabular}

Tabla 3.4. Madres transgénero y madres XY

\begin{tabular}{|l|l|l|l|}
$\begin{array}{c}\text { Autor/es, año } \\
\text { y país }\end{array}$ & $\begin{array}{c}\text { Diseño del } \\
\text { estudio }\end{array}$ & \multicolumn{2}{c}{ Método de inducción } \\
$\begin{array}{l}\text { Reisman et al. } \\
(2018) \text { EEUU }\end{array}$ & $\begin{array}{l}\text { Estudio de } \\
\text { un caso }\end{array}$ & $\begin{array}{l}\text { Domperidona }+ \\
\text { progesterona }+ \\
\text { estradiol + sacaleches }\end{array}$ & $\begin{array}{l}\text { Satisfactorio. Madre transgénero } \\
\text { consigue lactancia exclusiva y mixta } \\
\text { para su hijo adoptivo }\end{array}$ \\
\hline $\begin{array}{l}\text { LeCain et al. } \\
(2020)\end{array}$ & $\begin{array}{l}\text { Estudio de } \\
\text { Reino Unido }\end{array}$ & $\begin{array}{l}\text { Domperidona }+ \\
\text { estrógeno únicamente } \\
+ \text { sacaleches }\end{array}$ & $\begin{array}{l}\text { Satisfactorio. Madre con cariotipo } \\
\text { XY y síndrome de insensibilidad a } \\
\text { los andrógenos consigue lactancia } \\
\text { mixta para su hijo gestado por }\end{array}$ \\
\hline
\end{tabular}


Una vez expuestos estos resultados de cada estudio seleccionado, se puede decir que la inducción a la lactancia es posible y tuvo lugar en todos los casos expuestos. Concretamente, se logró lactancia exclusiva en cuatro de los dieciséis artículos (Abejide et al. 1997; McGuire, 2019; Nemba, 1994; Szucs et al., 2010;), mixta en siete de dieciséis (Auerbach et al., 1981; Biervliet et al., 2001; Farhadi et al., 2017; LeCain et al., 2020; Shiva et al., 2010; Wilson et al. 2015; Zingler et al. 2017;), mujeres con exclusiva y mixta en tres (Banapurmath et al., 1993; Cheales-Siebenaler, 1999; Reisman et al., 2018) y finalmente un caso donde la mujer se convirtió además en donante de leche (Flores-Antón et al., 2017).

\section{DISCUSIÓN}

Con respecto a la experiencia publicada de casos de inducción a la lactancia, la inmensa mayoría de mujeres consiguen poder dar el pecho. A lo largo de la literatura, concretamente en los inicios sobre el conocimiento acerca del tema, se han usado los términos «inducción»y «relactación» indiscriminadamente, por lo que en algunos artículos las mujeres ya habían estado embarazadas o habían lactado previamente, en cuyo caso, el término más apropiado sería el de «relactación». En el estudio de Abejide et al. (1997), las participantes consiguen lactancia exclusiva sin usar fármacos y coinciden que todas habían gestado y habían amamantado con anterioridad. Este tipo de mujeres que habían dado el pecho previamente, en el estudio de Auerbach (1981) conseguían leche más fácil y rápidamente, atendiendo este hecho a la posibilidad de creación de células memoria en una primera gestación (Dos Santos et al., 2015), como se ha comentado con anterioridad.

No obstante, en el estudio de Banapurmath et al. (1993), a pesar de que las participantes también habían tenido embarazo y lactancia previas, unas consiguen lactancia exclusiva y el resto mixta. En el caso del estudio de Nemba (1994), tanto las mujeres que inducen como las que relactan produjeron la leche de igual forma. ChealesSiebenaler (1999) y Szucks (2010) relatan los casos de inducciones en madres adoptivas que consiguen lactancia exclusiva, de igual modo que Flores-Antón et al. (2017) describe a una madre adoptiva que logra lactancia exclusiva y convertirse en donante de leche. En estos tres últimos descritos, ninguna había realizado lactancia materna previa. 
Por todo ello, la lactancia previa es un factor fuertemente influyente pero no determinante del éxito de la lactancia, puesto que todas las mujeres tienen la misma capacidad.

En lo que a métodos se refiere, se describen tanto farmacológicos como no farmacológicos. Cabe mencionar que existe una diferencia entre los casos descritos de «relactación»e «inducción», y es que para la inducción se necesita la combinación de ambos métodos, no siendo esto un hecho estrictamente necesario en la relactación, donde pueden ser suficientes los métodos no farmacológicos (Abejide, 1997; Auerbach, 1981). De todas formas, en todos ellos hay un denominador común que es clave: la estimulación del pecho y pezón (Abejide, 1997; Auerbach, 1981; Flores-Antón et al., 2017; Nemba, 1994; Szucs et al., 2010; Wilson et al., 2015). La estimulación puede ser mediante sacaleches eléctrico (preferiblemente doble), extracción manual y por la succión del bebé. Otros factores descritos que promueven el éxito son la motivación de la madre y el apoyo emocional de su familia y personal sanitario (Abejide, 1997; Nemba, 1994; Szucks et al., 2010).

La lactancia mixta con el uso de suplementación es un hecho frecuente al que las madres que se inducen recurren, debido a una producción insuficiente como para amamantar de forma exclusiva en algunas madres. No obstante, no es un hecho que les repercuta de forma negativa ya que, para la mayoría de ellas, el objetivo primordial es la unión emocional con su bebé (Auerbach, 1981; Biervliet et al. 2001; Farhadi y Philip, 2017; Flores-Antón et al., 2017; LeCain et al., 2020; Szucs et al., 2010; Wahlert y Fiester, 2013; Wilson et al., 2015), a excepción del estudio de Nemba (1994) y Abejide (1997), donde el objetivo es la supervivencia de los bebés dadas las precarias circunstancias.

Con todo lo expuesto, surge la necesidad de definir qué es una lactancia inducida de éxito, dado que será de éxito o no en función de los objetivos y expectativas iniciales de la madre que, como se ha relatado a lo largo del trabajo, pueden ser nutricionales, emocionales, un factor de supervivencia o la producción mayor o menor de leche. Por tanto, antes de iniciar el proceso cabría puntualizar esta cuestión. La Figura 3 presenta un algoritmo sobre el éxito de la inducción donde se presentan dos escenarios: una inducción exitosa o, por el contrario, una inducción fallida, y se exponen los factores que han podido llevar al fracaso, siendo los dos factores clave la madre y el bebé. 
Finalmente, en estos últimos años se han publicado casos de madres que distan a los modelos descritos a lo largo de la literatura. En el estudio de Reisman, Goldstein et al. (2018), una madre transgénero logra dar el pecho, a través de la inducción, de forma exclusiva durante 6 semanas a su hijo adoptado. Seis años atrás empezó su feminización hormonal y para el momento en el que se plateó la adopción e inducción tomaba estradiol, progesterona y espironolactona, esta última para el bloqueo androgénico. Este régimen hormonal se mantuvo para la inducción incrementando gradualmente las dosis $\mathrm{y}$ añadiéndose Domperidona junto con la estimulación mediante sacaleches. El bloqueo androgénico para la inducción en estas mujeres es necesario y se mantuvo durante la lactancia. En el estudio de LeCain et al. (2020) se describe a una mujer con fenotipo femenino y con cariotipo XY que padece el síndrome de insensibilidad a los andrógenos. Es diagnosticada en la infancia, se le extirpan ambas gónadas y se le pauta sustitución hormonal con estrógenos en su adolescencia. En su proceso inductivo se mantienen estos estrógenos y se incorpora la Domperidona y la estimulación del pecho con sacaleches, consiguiendo una lactancia mixta para su hijo gestado por sustitución. No se ha descrito otro caso como este en el que la inducción se lleve a cabo mediante únicamente estrógenos, lo que lleva a sugerir por parte de los autores que el uso de la progesterona podría no sería esencial para la lactogénesis.

Estos dos últimos artículos marcan una diferencia con respecto al resto de las mujeres y protocolos usados en el resto de las publicaciones, pero no por ello muestran una inferioridad o ineficacia en el proceso, dado que son igualmente capaces de dar el pecho a sus bebés. Ambos ejemplifican la no necesidad de tener órganos reproductores femeninos para poder amamantar, como un útero u ovarios, o un cariotipo XX. Todo ello me lleva a reflexionar si la lactancia masculina (Lafont, 2015) podría ser posible en un futuro como una realidad y posibilidad más de poder amamantar, de manera que la inducción permitiese un rol de lactancia compartido entre madre y padre, puesto que, a grandes rasgos, solo necesitaríamos un adecuado protocolo hormonal (incluyendo el bloqueo androgénico), farmacológico y de estimulación junto con una hipófisis normofuncionante.

\section{CONCLUSIONES}

En nuestra sociedad actual, van apareciendo cada vez más nuevos conceptos de familia distintos al modelo «tradicional» de padre y madre, como serían parejas homosexuales, 
parejas monoparentales y parejas que recurren a las técnicas de reproducción asistida, adopción o gestación por sustitución. Todas estas circunstancias, inclusive madres transgénero o madres cariotípicamente $\mathrm{XY}$, como se ha podido ver a lo largo de la revisión, sientan las bases de futuros posibles escenarios cada vez más frecuentes donde la opción de inducir la lactancia debe estar presente, dándola a conocer y dando a conocer a su vez que, a pesar de no ser un proceso sencillo y rápido, y que requiere de una elevada motivación personal y apoyo emocional, es factible y proporciona una fuente de múltiples beneficios bidireccionales para madre e hijo. Estos beneficios abarcan los inmunológicos, nutricionales y emocionales, promoviendo la unión y vínculo maternofilial y, a su vez, el empoderamiento de madres no biológicas, pero madres, al fin y al cabo.

De este modo, para poder promover esta alternativa a las madres, es esencial que los profesionales sanitarios estén informados y formados acerca de esta cuestión, y que formen parte del pilar fundamental de apoyo antes, durante y después del proceso de inducción. Con todo ello, se requiere de más estudios con mayor grado de calidad de evidencia que permitan la estandarización y comparación de métodos con el fin de tener una guía específica, útil y práctica para cada caso en particular, y que permitan poder proporcionar más información acerca de la seguridad y eficacia de los fármacos y fitoterapia empleados.

Unificando las técnicas y protocolos existentes, se han creado dos algoritmos (Figura 2 y 3), con la finalidad de poder sentar una base a través de la cual profesionales sanitarios y consultoras de lactancia sean capaces de orientar, personificar e individualizar la inducción según las circunstancias de la madre y del bebé. El primer algoritmo (Figura 2) describe los pasos a seguir para conseguir la inducción a la lactancia, y el segundo protocolo (Figura 3) serviría para el control evolutivo de esta, así como también para proporcionar herramientas en caso de inducción no satisfactoria o en caso de necesitar refuerzo con suplementación. 
Figura 2. Algoritmo sobre la lactancia materna inducida. Las estrellas sirven para unir cajas que tienen el mismo número de estrellas, quedando así vinculadas. Las cajas con una estrella se relacionan entre sí, y lo mismo sucede entre las cajas con dos estrellas, y así sucesivamente. Fuente: elaboración propia.

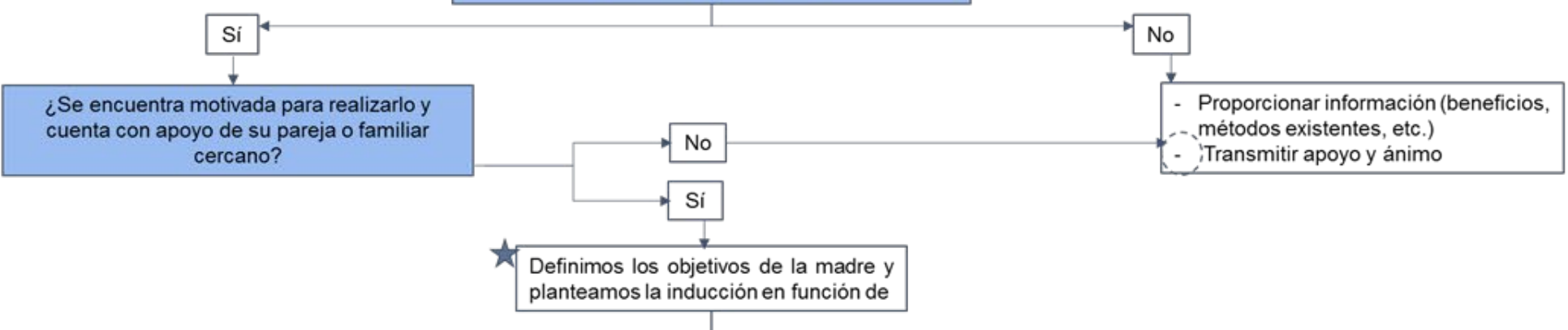

ACO: anticonceptivos orales 1/35 $\geq 1 \mathrm{mg}$ progesterona $0,035 \mathrm{mg}$ estrógenos

Administracion: solo plidora

$$
\text { ACO + Galactogógos }
$$

\section{Madre cisgénero: ACO 1/35}

Madre transgénero: añadir bloqueo androgénico con antagonistas aldosterona (ej.: espironolactona)

Madre $X Y$ con Sindrome insensibilidad a andrógenos: se podria llevar a cabo a andrógenos: se podria llevar a cab
la inducción sólo con estrógenos

Madre no tolera ACO: probar

alternativas tópicas (cremas, parches...) Si ACO contraindicados (por trombosis, patologia cardiaca, hipertensión inductivas o probar el uso concomitante de ACO con Metildopa

\begin{tabular}{|l|}
\hline Antagonistas \\
dopaminérgicos \\
-Domperidona: de \\
elección. \\
-Otros: \\
Metoclopramida \\
\hline
\end{tabular}

Tipo de inducción que se desea

\begin{tabular}{|l|l|}
\hline $\begin{array}{l}\text { Masajes + } \\
\text { extracciones con } \\
\text { sacaleches } \\
\text { manual o } \\
\text { eléctrico } \\
\text { (preferiblemente } \\
\text { doble) }\end{array}$ & $\begin{array}{l}\text { No fenogreco } \\
\text {-Cardo Bendito }\end{array}$ \\
\hline
\end{tabular}

\begin{tabular}{|c|}
\hline Combinada \\
\hline $\begin{array}{c}\text { Farmacológica }+ \\
\text { no farmacológica } \\
\text { Se recoge su uso } \\
\text { en los diferentes } \\
\text { protocolos }\end{array}$ \\
\hline
\end{tabular}

En casos de relactación: podrian usarse únicamente métodos no farmacológicos

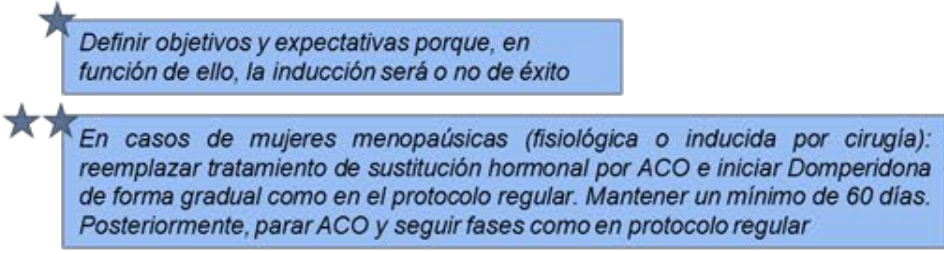

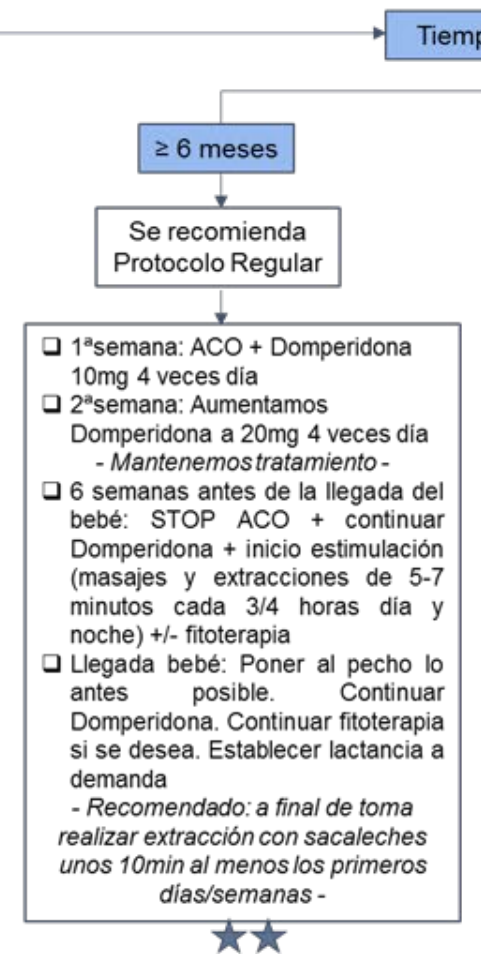

Tiempo disponible antes de la llegada del bebé 


\section{$\mathrm{Si}$}

- Continuar lactancia

Nalorar si se necesita uso de suplementación

Si lactancia bien establecida valorar la bajada

de dosis de Domperidona (de 20 a 10mg) o

su posible retirada definitiva de forma gradual

Mantener controles
- Pediátricos habituales (control ganancia peso,
orina y deposiciones, actividad)

Ginecológicos/Obstétricos + consultoras lactancia

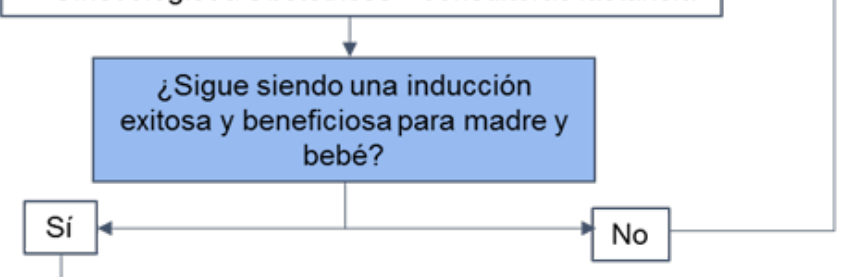

$\checkmark$

- Refuerzo positivo

- Mantener lactancia hasta

el deseo de su cese (La

el deseo de su cese (La
OMS recomienda lactancia

materna mínimo los 6 primeros

meses de vida)

Según la Guia de Lactancia Materna de la AEP ${ }^{24}$, los criterios principales de buen inicio y buena instauración de la lactancia son:

- Buena postura, buen agarre

$\checkmark$ Cabeza frente al pecho. Pezón altura nariz. Cuello ligeramente deflexionado.

$\checkmark$ Barbilla toca pecho. Nariz libre

$\checkmark$ Boca abierta, labio inferior evertido. Ángulo labios de unos $120^{\circ}$

$\checkmark$ Menos areola visible por debajo de la barbilla que por encima del pezón

Madre no dolor. Pezón no se le escapa al bebé

- Madre y bebé cómodos. Bebé mama tranquilo. Realiza succiones profundas y mantenidas $y$ muestra signos de saciedad al final de la toma

- Aumento del volumen de leche

Bebé muestra buenos signos de hidratación, disposición a mamar (mínimo 8 veces/dia), micciones, deposiciones y ganancia de peso adecuadas.

- Madre siente seguridad y confianza en si misma. Detecta las señales del bebé cuando éste está dispuesto a mamary ofrece el pecho para ello

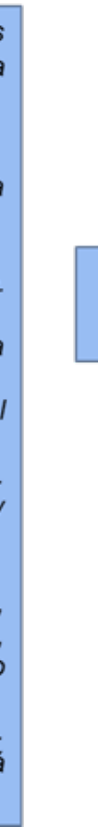




\section{AGRADECIMIENTOS}

Agradecemos a Alba Padró (cofundadora de LactApp, y presidenta de ALBA Lactancia) por orientarnos en cómo enfocar toda la información. A Gemma Cazorla (comadrona) por facilitarnos bibliografía y experiencias sobre el tema. Y a Eva Beade (asesora en lactancia) por ofrecernos asesoría sobre la lactancia. 


\section{BIBLIOGRAFÍA}

ABEJIDE, O., et al. Non-Puerperal Induced Lactation in a Nigerian Community: Case Reports. En: Annals of Tropical Paediatrics. 1997, vol. 17, núm. 2, pp. 109-114.

AFFLERBACK, S., et al. Infant-feeding consumerism in the age of intensive mothering and risk society. En: Journal of Consumer Culture. 2013, vol. 13, pp. 387-405.

APILAM - ASOCIACIÓN PARA LA PROMOCIÓN E INVESTIGACIÓN CIENTÍFICA Y CULTURAL DE LA LACTANCIA MATERNA. 2002. Disponible en: http://e-lactancia.org

Auerbach, K. G. Induced Lactation A Study of Adoptive Nursing by 240 Women. En: American Journal of Diseases of Children. 1981, vol. 135, pp. 340-43.

BANAPURMath, C. R., et al. Successful Induced Non-Puerperal Lactation in Surrogate Mothers. En: The Indian Journal of Pediatrics. 1993, vol. 60, pp. 639-643

BIERVLIET, F. P., et al. Induction of Lactation in the Intended Mother of a Surrogate Pregnancy. En: Human Reproduction. 2001, vol. 16, núm. 3, pp. 581-583.

BOWLBY, John. Una base segura: aplicaciones clínicas de una teoría del apego. Buenos Aires: Paidós, 1989.

CAppellini, B., et al. Intensive mothering in hard times: Foucauldian ethical selfformation and cruel optimism. En: Journal of Consumer Culture. 2019, vol. 19, núm. 4, pp. 469-492.

CAzOrla OrTiz, G. Métodos no farmacológicos para inducir la lactancia materna en adopción, subrogación y en la madre no gestante en parejas lesbianas: Revisión bibliográfica. En: MUSAS: Revista de Investigación En Mujer, Salud y Sociedad. 2018, vol. 3, núm. 1.

CAZOrla-Ortiz, G., et al. Understanding the Challenges of Induction of Lactation and

Relactation for Non-Gestating Spanish Mothers. En: Journal of Human Lactation. 2019, vol. 36, núm. 3, pp. 528-536.

CheAles-Siebenaler, N. J. Induced Lactation in an Adoptive Mother. En: Journal of Human Lactation, 1999, vol. 15, núm. 1, pp. 41-43.

Dos Santos, Camila O., et al. An Epigenetic Memory of Pregnancy in the Mouse Mammary Gland. En: Cell Reports. 2015, vol. 11, núm. 7, pp. 1102-1109.

FARHADI, R.; PHILIP, R. K. Induction of Lactation in the Biological Mother After Gestational Surrogacy of Twins: A Novel Approach and Review of Literature. En: Breastfeeding Medicine. 2017, vol. 12, núm. 6, pp. 373-376.

Flores-Antón, B.; GarcíA-Lara, N. R.; PAllás-Alonso, C. R. An Adoptive Mother Who Became a Human Milk Donor. En: Journal of Human Lactation. 2017, vol. 33, núm. 2, pp. 419-421.

Goldfarb, L.; Newman, J. Introduction to the Protocols for Induced Lactation. Canadian Breastfeeding Foundation. Fondation canadienne de l'allaitement, 2002. Disponible en: https://bit.ly/3KlyuIE. 
GribBLE, Karleen D. The Influence of Context in the Success of Adoptive Breastfeeding: Developing Countries ant the West. En: Breastfeeding review. 2004, vol. 12, núm. 1, pp. 5-13.

GribBLE, Karleen D. A Model for Caregiving of Adopted Children After Institutionalization. En: Journal of Child and Adolescent Psychiatric Nursing. 2007, vol. 20, núm. 1, pp. 14-26.

HARrison, Tinsley Randolph. Harrison. Principios de Medicina interna, vol. 1 y 2, editado por Kurt J. Isselbacher, et al. Nueva York: McGraw-Hill Interamericana, 2018.

LAFONT, Román. Lactancia masculina inducida. En: Revista Cubana de Pediatría. 2015, vol. 87, núm. 4, pp. 487-498.

Lecain, M.; Fraterrigo, G.; Drake, W. M. Induced Lactation in a Mother Through Surrogacy with Complete Androgen Insensitivity Syndrome (CAIS). En: Journal of Human Lactation. 2020, vol. 36, núm. 4, pp. 791-794.

Marshall, J. L.; Godfrey, M.; Renfrew, M. J. Being a «good mother»: Managing breastfeeding and merging identities. En: Social Science \& Medicine. 2007, vol. 65, núm. 10, pp. 2147-2159.

MCGUIRE, E. Induced lactation and mothers sharing breastfeeding: A case report. En: Breastfeeding Review. 2019, vol. 27, núm. 2, pp. 37-41.

Ministerio de Sanidad, Servicios Sociales e Igualdad. Guía de Práctica Clínica sobre lactancia materna. 2017. Disponible en: https://www.aeped.es/sites/default/files/guia de lactancia materna.pdf

Ministerio De SANidAd, Servicios Sociales E IguAldad. Informe anual del Sistema Nacional de Salud. 2018. Disponible en: https://bit.ly/3GyhGvz.

Mortel, M.; MeHTA, S. D. Systematic Review of the Efficacy of Herbal Galactogogues. En: Journal of Human Lactation. 2013, vol. 29, núm. 2, pp. 154-162.

NemBA, K. Induced Lactation: A Study of 37 Non-Puerperal Mothers. En: Journal of Tropical Pediatrics. 1994, vol. 40, pp. 240-242.

Ogunlesi, T. A., et al. Non-Puerperal Induced Lactation: An Infant Feeding Option in Paediatric HIV/AIDS in Tropical Africa. En: Journal of Child Health Care. 2008, vol. 12, núm. 3, pp. 241-248.

OMS - ORGANIZACIÓN MUNDIAL DE LA SALUD. Relactation: Review of experience and recommendations for practice. 1998. Disponible en: https://bit.ly/3nupEhO.

OMS - ORgANizACiÓN Mundial DE LA SALud. 10 facts on breastfeeding. 2017. Disponible en: http://www.who.int/features/factfiles/breastfeeding/es/.

Perrin, M., et al. A Pilot Study on the Protein Composition of Induced Nonpuerperal Human Milk. En: Journal of Human Lactation. 2014, vol. 31, núm. 1, pp. 166-171.

ReISMAn, T.; Goldstein, Z. Case Report: Induced Lactation in a Transgender Woman. En: Transgender Health. 2018, vol. 3, núm. 1, pp. 24-26. 
SHIVA, M., et al. A Successful of Lactation in Surrogate Pregnancy with Metoclopramide and Review of Lactation Induction. En: International Journal of Fertility and Sterility. 2010, vol. 3, núm. 4, pp. 191-194.

SzUCS, K., et al. Induced Lactation and Exclusive Breast Milk Feeding of Adopted Premature Twins. En: Journal of Human Lactation. 2010, vol. 26, núm. 309-313.

WAHLERT, L.; FIESTER, A. Induced Lactation for the Nongestating Mother in a Lesbian Couple. En: Virtual Mentor. 2013, vol. 15, núm. 9, pp. 753-756.

WiLliams, K., et al. Discursive constructions of infant feeding: The dilemma of mothers' «guilt». En: Feminism \& Psychology. 2012, vol. 23, núm. 3, pp. 339-358.

WiLsOn, E., et al. The Intricacies of Induced Lactation for Same-Sex Mothers of an Adopted Child. En: Journal of Human Lactation. 2014, vol. 31, núm. 1, pp. 64-67.

ZINGLER, E., et al. Lactation Induction in a Commissioned Mother by Surrogacy: Effects on Prolactin Levels, Milk Secretion and Mother Satisfaction. En: Revista Brasileira de Ginecologia e Obstetrícia / RBGO Gynecology and Obstetrics. 2017, vol. 39, núm. 2, pp. 86-89. 